DOI: $10.6060 / \mathrm{mhc} 190229 \mathrm{k}$

\title{
Molecular Structure and Conformation of Free Base Corroles
}

\author{
Mikalai M. Kruk, ${ }^{a @ 1}$ Dmitry V. Klenitsky,a and Wouter Maes ${ }^{\mathrm{b} @ 2}$ \\ Dedicated to the 85-th anniversary of Academician G. P. Gurinovich \\ ${ }^{a}$ Belarusian State Technological University, Physics Department, 220006 Minsk, Belarus \\ ${ }^{\mathrm{b}}$ Design \& Synthesis of Organic Semiconductors (DSOS), Institute for Materials Research (IMO-IMOMEC), Hasselt \\ University, B-3590 Diepenbeek, Belgium \\ ${ }^{@ 1}$ CorrespondingauthorE-mail: krukmikalai@yahoo.com;m.kruk@belstu.by \\ ${ }^{\circledR 2}$ Corresponding authorE-mail: wouter.maes@uhasselt.be
}

\begin{abstract}
Corroles represent a unique family of tetrapyrrolic chromophores with distinct structural, chemical and photophysical properties, which render them promising materials for numerous applications. The lack of one of the meso-carbon atoms ultimately leads to nonplanar macrocycle distortions. The aim of this review is to summarize the peculiarities of the molecular conformations of free base corroles and provide a unified approach for their analysis.
\end{abstract}

Keywords: Free base corroles, macrocycle, mean plane, substitution pattern, nonplanar distortions, conformers.

\section{Молекулярная структура и конформация свободных оснований корролов}

\author{
Н. Н. Крук, ${ }^{a} 11$ А. В. Кленицкий, ${ }^{a}$ B. Маес ${ }^{\mathrm{b}}{ }^{2}$ \\ Посвящается 85-летию со дня рождения академика Г. П. Гуриновича

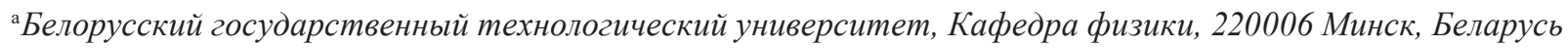 \\ b Лаборатория дизайна и синтеза органических полупроводников, Институт исследования материалов, \\ Университет Хассельта, В-3590 Дипенбек, Бельгия \\ @1E-mail:krukmikalai@yahoo.com;m.kruk@belstu.by \\ @2E-mail: wouter.maes@uhasselt.be
}

\begin{abstract}
Корроль представляют собой уникальное семейство тетрапиррольных хромофоров с особенными структурными, химическими и фотофизическими свойствами, которые делают их перспективными соединениями для многочисленных применений. Отсутствие одного из мезо-атомов углерода неизбежно приводит к непланарным искажениям макроцикла. Целью данного обзора является обобщение сведений об особенностях молекулярной конформации свободных оснований корролов и формулировка единого подхода $\kappa$ их описанию.
\end{abstract}

Ключевые слова: Свободные основания корролов, макроцикл, средняя плоскость, архитектура замещения, непланарные искажения, конформеры. 


\section{Introduction}

The parent tetrapyrrolic macrocycle "porphine" is known to have four pyrrolic fragments connected through methine bridges. The tetrapyrrolic family also comprises compounds in which two adjacent rings (pyrrole and pyrrolenine) are bound directly through a $\mathrm{C}_{\mathrm{a}}-\mathrm{C}_{\mathrm{a}}$ bond, such as porphycenes and corroles. Whereas the former are structural isomers of porphine, the latter constitute the group of contracted tetrapyrrolic compounds lacking one meso-carbon atom (see Figure 1). For aromatic stabilization to occur, the contracted macrocycle must fulfill the Hückel $(4 n+2)$ rule for the number of $\pi$-electrons, which is maintained by the increase in the number of pyrrole rings at the expense of a pyrrolenine. Thus, the contracted corrole macrocycle consists of three pyrrole and one pyrrolenine ring rather than two pyrrole and two pyrrolenine rings in the standard free base (i.e. metal free) porphyrin molecules. Reduction of the core size due to the macrocycle's contraction and the increase in the pyrrole proton number (there are three protons in the core now) are two intrinsic features which ultimately promote nonplanar macrocycle distortions in free base corroles. As a result, the corrole macrocycle achieves a nonplanar conformation, even if sterical strains from peripheral substituents are absent. It is worth to note here that reducing the distance between the adjacent pyrrolic nitrogen atoms favors the formation of an intramolecular hydrogen NH...N bond, which acts as an opposing force to the forces promoting nonplanar macrocycle distortions.

Starting from the first paper dealing with the X-ray analysis of corroles, ${ }^{[1]}$ where it was found that the corrole macrocycle is not planar, all following works have confirmed this feature. However, upon analysis of the distortions, there is no consensus with respect to their quantification. Hence, a crucial question to be addressed by all people involved in corrole chemistry and spectroscopy is how to actually consider these nonplanar distortions, either using the established approach for porphyrin macrocycles or by accepting a new basis? Both ways obviously have advantages and disadvantages. Whereas the first approach allows to easily interpret the structural features of corrole macrocycles in one common way for all tetrapyrrolic macrocycle frameworks, the decrease in molecular symmetry for corroles does not allow direct use of the classification of nonplanar distortion types as developed for porphyrins. ${ }^{[2]}$ The archetype macrocycle structure for tetrapyrrolic compounds is the porphine, which has a planar conformation and no intrinsic steric hindrance in the macrocycle core. Therefore, all molecular conformations can be quantified by the type and magnitude of the deviations from planarity. Planarity is then defined by the macrocycle mean plane in which all skeletal atoms of the porphine macroring are situated. On the contrary, the archetype macrocycle for the corrole family has an intrinsically nonplanar molecular conformation (vide supra). Moreover, the asymmetry of the corrole macrocycle leads to distinctly different geometries for the two potential $\mathrm{NH}$ tautomers with unique arrangement of the pyrrole protons (Figures 1), which needs to be taken into account upon molecular conformation analysis.

Thus, the important set of questions that need to be answered when considering the particular molecular con-

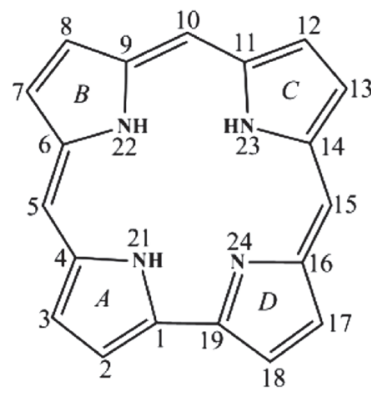

T1

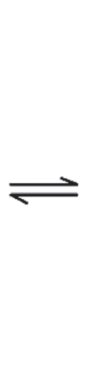

$\mathrm{T} 2$

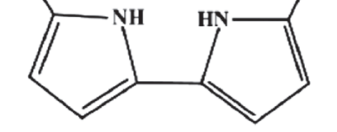

Figure 1. Molecular structures of the two tautomeric forms of free base corroles (with atom numbering according to IUPAC nomenclature). The NH tautomers are numbered as suggested in Refs..${ }^{[3,4]}$ where the tautomer showing the longest wavelength of the $0-0$ transition maximum was assigned as the $\mathrm{T} 1$ tautomer and that of higher energy to the T2 tautomer. Thus, the pyrrole rings $A$ and $B$ bear protons in both tautomeric forms, while rings $D$ and $C$ may form a hydrogen bond. Pyrrole rings $A$ and $D$ are bound directly through a $\mathrm{C}_{1}-\mathrm{C}_{19}$ bond.

formations of free base corroles are: i) How to evaluate the macrocycle distortions, either by comparison to an imaginary "mean" plane, or relative to the real conformation of the archetype macrocycle? ii) Which plane should be defined as the "mean" plane? iii) How to take into account the macrocycle asymmetry from the point of view of both the skeletal asymmetry and the formation of two corrole $\mathrm{NH}$ tautomers? To date, there is no consensus on the description and analysis of free base corrole macrocycle molecular conformations. Several distinguishing features of the corrole macrocycle have been pointed out, however, without characterization of the macrocycle geometry as a whole.

\section{Character of the Macrocycle Conformation and Search for a Proper Mean Macrocycle Plane}

First X-ray analysis data for corrole macrocycles (for 8,12-diethyl-2,3,7,13,17,18-hexamethylcorrole) indicated a deviation from the planar macrocycle conformation, which was attributed to the shortening of the N-N contacts in the macrocycle core as a result of the direct $\mathrm{C}_{1}-\mathrm{C}_{19}$ linkage. ${ }^{[1]}$ To quantify the distortions, a reference "mean" plane was chosen containing 31 atoms $(19 \mathrm{C}+4 \mathrm{~N}$ of the macrocycle and the 8 closest $\mathrm{C}$ atoms of the alkyl substituents). The obtained picture for the out-of-plane atom displacements is shown in Figure 2.

Indeed, the nonplanar distortions of the macrocycle and their asymmetry are well visible. At the same time the shortcoming of such a choice of a "mean" plane is also clear: only one skeletal atom lies in the "mean" plane. All four nitrogen atoms were found to bear protons. This means that either no exclusive stabilization of one $\mathrm{NH}$ tautomer takes place, or the X-ray diffraction analysis does not provide sufficient details. However, the proton occupation probabilities seem to be different since the geometries are different for all pyrrole rings. The T2 tautomer is preferentially populated (i.e. proton-free pyrrole $C$ ) and the $\mathrm{T} 1$ 


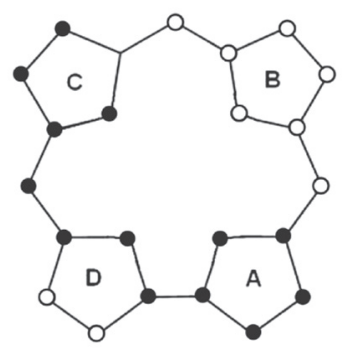

Figure 2. Out-of-plane atom displacements from the assigned "mean" plane for 8,12-diethyl-2,3,7,13,17,18-hexamethylcorrole based on the X-ray data as obtained from Ref. ${ }^{[1]}$

tautomer is less populated (i.e. proton-free pyrrole $D$ ). This conclusion is based on the findings presented in Ref., ${ }^{[5]}$ namely that the $\mathrm{C}_{\mathrm{a}}-\mathrm{N}-\mathrm{C}_{\mathrm{a}}$ angle in the proton-free pyrrole is distinctly smaller compared to that of the protonated ones. This pattern has been confirmed later on for both the X-ray and DFT-optimized geometries of several free base corroles. ${ }^{[4,6]}$ The planes of pyrroles $B, C$ and $D$ differ slightly, whereas pyrrole $A$ rotates for about $8-10^{\circ}$. A noticeable shortening of the N-N distances in the macrocycle core is observed, with the N(pyrrole $A$ )-N(pyrrole $D$ ) distance being the shortest $(2.54 \AA)$, which could be even shorter in a planar macrocycle conformation. Thus, this shortening can be considered as the first intrinsic strain leading to out-of-plane distortions. Two bonds from pyrrole $A\left(\mathrm{C}_{\mathrm{a}}-\mathrm{C}_{\mathrm{a}}\right.$ and $\left.\mathrm{C}_{\mathrm{a}}-\mathrm{C}_{\mathrm{m}}\right)$ are longer due to ring rotation, since it decreases their $\pi$-character. The molecule was found to be asymmetric in the crystal, giving rise to the possibility to adopt enantiomeric forms.

Later on, quantum-chemical geometry optimization of the unsubstituted free base corrole macrocycle has been done, while constraining to the macrocycle to be planar $\left(\mathrm{C}_{\mathrm{s}}\right.$ symmetry). ${ }^{[5]}$ The authors indicated that the relative flatness of the potential energy surfaces associated with the out-of-plane distortions makes accurate conformational analysis troublesome. Nevertheless, some conclusions have been drawn from the computed data. The macrocycle core is smaller compared to that in porphyrins, leading to shorter $\mathrm{N}-\mathrm{N}$ distances. The direct $\mathrm{C}_{\mathrm{a}}-\mathrm{C}_{\mathrm{a}}$ linkage was confirmed to be the principal source of strain in the molecular structure of corroles. The NH tautomers were shown to be quite close in energy. This fact, taken together with the short, strong NH...N hydrogen bonds within the core, lead to the assumption that $\mathrm{NH}$ tautomerization can occur considerably faster in corroles compared to porphyrins. The $\mathrm{C}_{\mathrm{a}}-\mathrm{C}_{\mathrm{a}}$ bond (1.41-1.42 $\AA$ ) was considerably longer than the $\mathrm{C}_{\mathrm{a}}-\mathrm{C}_{\mathrm{m}}$ bond (1.39-1.40 $\AA$ ). The $\mathrm{C}_{\mathrm{a}}-\mathrm{N}-\mathrm{C}_{\mathrm{a}}$ angles in the protonated pyrrole rings were significantly larger $\left(111.5-114.3^{\circ}\right)$ than those in the non-protonated pyrrole rings $\left(106.5-107.8^{\circ}\right)$. This trend parallels with the one observed in porphyrins. Steric repulsion of the central nitrogen atoms and NH...N hydrogen bonding can hence be concluded to be the source of the second intrinsic strain leading to substantial differences in the $\mathrm{N}-\mathrm{C}_{\mathrm{a}}-\mathrm{C}_{\mathrm{m}}$ angles in both corrole $\mathrm{NH}$ tautomers and corrole/porphyrin pairs. The angle values span a large range from 116.5 to $129^{\circ}$.

The role of solvent molecules in the stabilization of a given macrocycle conformation has been confirmed through X-ray data by Gross et al. ${ }^{[7]}$ It was demonstrated that the solvent molecules (EtOH or $m$-xylene) are involved in hydrogen-bonding interactions with the imine-like nitrogen atoms. This paper reported on the molecular structure of 5,10,15-tris-pentafluorophenylcorrole (likely the T2 tautomer), but the data quality was not sufficient to assign the positions of the core protons unambiguously. The term "puckered" was used to describe the character of the nonplanar conformer. Puckering is considered as a conformation where the pyrroles are turned slightly up or down. No order in the direction of this turn is pointed out ("The twist angles between the rings increase moving clockwise from $B$ around the macrocycle in the order of 4.4 , $9.4,19.1$, and $19.5^{\circ}$, respectively"). ${ }^{[7]}$ The authors mentioned that the distortions observed provide the shortest possible van der Waals distances for hydrogen atoms.

For 5,10,15-tris-heptafluoropropylcorrole, only the T1 tautomer was observed in the crystals. ${ }^{[8]}$ However, the authors stress their expectation that this tautomer is not the most stable one, referring to the data from Ref. ${ }^{[5]}$ However, in Ref., ${ }^{[5]}$ the overall macrocycle planarity was fixed in the computational procedure and the presented value of $2.45 \mathrm{kcal} / \mathrm{mol}$ for the ground state energy difference between the two $\mathrm{NH}$ tautomers seems not be used for the argumentation. Moreover, the computed molecule is the unsubstituted archetype corrole macrocycle rather than a tris-meso-alkylated or tris-meso-arylated derivative, i.e. additional steric hindrance arising from the meso-substituents is neglected. The "mean" plane of the four nitrogen atoms (4N plane) was introduced to quantify the nonplanar distortions. ${ }^{[8]}$ The importance of steric hindrance of the three inner protons for the formation of the nonplanar conformation with the pyrroles turned alternatively up and down was underlined. Again, the twist angles between the rings were applied. Clockwise, starting from $B$, the angles are 20.2, 11.6, 13.9 and $18.0^{\circ}$. Two conclusions can be drawn from comparison of these data for the T1 tautomer with the corresponding data set for the T2 tautomer taken from Ref. ${ }^{[7]}$ First, the out-of-plane distortions in the T1 tautomer have a higher magnitude compared to the T2 tautomer. Second, the substitution pattern has a strong influence on the equilibrium between the two tautomers and preferential stabilization (if any) upon crystal growth. In the framework of the $4 \mathrm{~N}$ mean plane, the positions of the three protons are discussed (in pyrroles $A$ and $B$, the hydrogen atoms are out-of-plane, while in pyrrole $C$ it is almost in-plane). Then, the respective deviations of the protons from the plane of their own pyrrole are reported. For the first time, the significant out-of-plane (pyrrole) displacement of the protons (in other words, the $\mathrm{N}$ atom "pyramidalization", i.e. $\mathrm{N}$ atoms acquiring some $s p^{3}$ character instead of pure planar $s p^{2}$ hybridization) has been noticed and the degree of displacement has been related to the corrole acidity. An important conclusion has been drawn relating the larger extent of pyramidalization to the higher acidity of a given corrole. The main structural changes upon going from the free base corrole to the Ga(III)complex (ligated with Pyr) were listed as follows. The twist angle of the pyrroles decreases: clockwise, starting from pyrrole $B$, the angles are $7.7,9.9,4.0$ and $7.0^{\circ}$. The mean deviation of the outer $\mathrm{C}_{b}-\mathrm{C}_{\mathrm{b}}$ bonds with respect to the $4 \mathrm{~N}$ plane decreases about 2-3 times. The $\mathrm{Ga}$ (III) ion is displaced 
for $0.31 \AA$ from the $4 \mathrm{~N}$ plane, i.e. using modern terminology one can say that a dome-type conformer is formed.

The $\mathrm{N}_{21}$ (pyrrole $A$ ) and $\mathrm{N}_{22}$ (pyrrole $B$ ) benzyl and picolyl substituted derivatives of 5,10,15-tris-pentafluorophenylcorrole have also been crystallized and studied by X-ray analysis. ${ }^{[9]}$ Most of the structural aspects were analyzed considering the torsion angles between the adjacent pyrroles, but a "mean" plane determined by the three nitrogen atoms of the unsubstituted pyrroles ( $3 \mathrm{~N}$ plane) was used when discussing the out-of-plane deviations of the core hydrogens. This choice stresses the asymmetry of the molecular conformation of these derivatives. Pyrrole $B$ substitution leads to more pronounced nonplanar distortions compared to pyrrole $A$ substituted derivatives. The pattern of the distortion enables to trace similarity between the $\mathrm{N}_{21}$ (pyrrole $A$ ) derivative and the T2 tautomer of the unsubstituted corrole, and between the $\mathrm{N}_{22}$ (pyrrole $B$ ) derivative and the T1 tautomer of the unsubstituted corrole. Such a spectral comparison was used successfully in Ref. ${ }^{[3]}$ for absorption spectra assignment to a particular configuration of core protons of two corrole NH tautomers. One can suggest that the NH...N hydrogen bond is retained upon $N$-substitution, and it acts to keep the conformers as planar as possible.

The T1 tautomer of 5,10,15-triphenylcorrole was also characterized in a crystal structure by Smith and co-workers in 2003. ${ }^{[10]}$ For the first time, it was attempted to analyze the macrocycle conformation as a whole using the concept developed for porphyrin macrocycles. Thus, the conformation of the free base 5,10,15-triphenylcorrole was suggested to be of the corrole saddle type, very similar to that in porphyrins. The mean deviation from the $(19 \mathrm{C}+4 \mathrm{~N})$ plane was $0.154 \AA$. The authors also analyzed the distortion pattern for several other $N$-substituted free base corroles, whose X-ray data were available at the time. The macrocycle conformations of these corroles were determined to be either corrole wave or corrole saddle/wave hybrid distortions, similar to the nonplanar porphyrin wave and saddle/ wave conformations. It was concluded that the corrole wave and corrole dome conformers are common (the former for free base corroles and the latter for the metallocorrole derivatives) and the corrole saddle conformer is less frequently seen.

For the 3-formyl substituted triphenylcorrole (T2 tautomer), the mean deviation of atoms from the $(19 \mathrm{C}+4 \mathrm{~N})$ plane was $0.152 \AA$, indicating that 3 -formyl substitution does not affect the macrocycle conformation. ${ }^{[1]}$ An almost coplanar configuration was observed for pyrroles $C$ and $D$, resulting from intramolecular NH...N hydrogen bonding. The 11-atom plane consisting of the 11 carbon atoms $\left(\mathrm{C}_{\mathrm{a}}\right.$ and $\left.\mathrm{C}_{\mathrm{m}}\right)$ of the macrocycle was also used for analysis. The dihedral angle between pyrrole $A$ and the 11 -atom plane is $15.0^{\circ}$, and that for pyrrole $B$ is $15.6^{\circ}$, but the tilt is in the opposite direction. As a result, the mutual dihedral angle between these two pyrrole planes is as large as $20.8^{\circ}$.

Preferred stabilization of the T2 tautomer of 5,10,15-tris-pentafluorophenylcorrole was reported at low temperature by the Gross group in 2004. ${ }^{[12]}$ The pattern found in the NMR spectra has been suggested to be the result of sharing of one of the core protons between two pyrroles. ${ }^{1} \mathrm{H}$ NMR signals were assigned to protons inter- acting with two different nitrogen atoms belonging to pyrrole $B$ (strong bonding, $\mathrm{N}-\mathrm{H}$ ) and $C$ (weak bonding, $\mathrm{H}$... N). Increase in temperature lead to the increase of the NH tautomerization rate and the $\mathrm{T} 1$ tautomer was also observed.

After multiple attempts to obtain crystal structures where the internal proton positions could be assigned unambiguously, two distinct structures corresponding to T1 and T2 tautomers have finally been reported by Ziegler et al. in 2005. ${ }^{[13]}$ The T1 tautomer was observed for 5,10,15-triphenylcorrole. The authors stated that the 19C plane is deformed from planarity, but pyrroles $C$ and $D$ are the closest to being coplanar and can be used to define the mean plane of the macrocycle. Tilting of pyrroles $A$ and $B$ in opposite directions out-of-(mean) plane was noticed. This structure does not conform to any of the four porphyrin conformations (see Ref. ${ }^{[2]}$ ). The same structural features have been revealed for the free base form of undecasubstituted 5,10,15-tris-pentafluorophenyl-2,3,7,8,12,13,17,18-octakis(4methoxyphenyl)corrole. ${ }^{[14]}$ The $C$ and $D$ pyrrole rings are the most coplanar (dihedral angle is $4.33^{\circ}$ only) and are used to define the mean plane of the macrocycle. The pyrroles $A$ and $B$ are tilted in opposite directions with the angle values are close to those found for the 5,10,15-triphenylcorrole. ${ }^{[13]}$

On the other hand, the T2 tautomer was observed for 5,10,15-tris-heptafluoropropylcorrole. A solvent molecule (ethyl acetate) was found in the crystal, hydrogen-bonded with the imine-like nitrogen atoms. This fact stresses the importance of solvation in the stabilization of the macrocycle conformation. The authors stated that pyrroles $B$ and $C$ are nearly parallel to the $19 \mathrm{C}$ plane and can be used to define the mean plane of the macrocycle. Tilting of pyrroles $A$ and $D$ in opposite directions out-of-(mean) plane was noticed and the conclusion is again that the structure of the T2 tautomer does not conform to any porphyrin conformation. It should be noted here that the mean plane is defined in a different way for the two NH tautomers, which is a clear shortcoming in the structural data analysis since the two tautomer structures cannot properly be compared.

Isocorroles have one or more reduced methine bridges (i.e. with $s p^{3}$-hybridized $\mathrm{C}_{\mathrm{m}}$ ). Due to this feature the isocorrole core contains only two protons. These molecules can nevertheless be interesting from the point of view of structural comparison with free base corroles. X-ray analysis data indicate that both the 5-isocorrole and two 10 -isocoroles are (nearly) planar. ${ }^{[15]}$ One needs to take into account that the 5-isororrole core is similar in size to that in corrole: the N-N distances are 2.58, 2.63, 2.65 and $3.00 \AA$ versus $2.90,2.78,2,64$ and $2.67 \AA$ with hydrogen bonds or 2.91 , 2.62, 2.61 and $2.71 \AA$ without (in 5,10,15-tris-pentafluorophenylcorrole). Therefore, the suggestion can be made that the repulsive interactions of the three protons are the main source of intrinsic strain imposed to the corrole macrocycle. We should also mention that flattening of the macroring is observed as well for the confused corrole derivatives (with NNNC core due to flipping of one of the pyrrole rings, i.e. exposure of the pyrrole $\mathrm{N}$ atom out of the macrocycle), which have two protons in the macrocycle core. ${ }^{[16]}$ The macrocycle is almost planar with exception of the flipped ring, which is tilted out of plane. The "flattened saddle conformation" reported for 3-nitro-5-hydroxy-isocorrole is likely to depend on the interactions of these two substituents (especially 
the outer hydrogen bonding that is possible between them).

Both X-ray data and TDDFT calculations are reported for a series of mono- and di-nitro-substituted tritolylcorroles by Paolesse et al., with special attention for the T1 tautomer of 2-nitro-5,10,15-tritolylcorrole. ${ }^{[6]}$ The structure of the latter compound was analyzed in detail. Thus, the dihedral angles between pyrroles $D$ and $C, A$ and $B$ are 10.5 and $23.8^{\circ}$, respectively. The twist between pyrroles $A$ and $D$ is $7.6^{\circ}$. The $\mathrm{N}$ atoms of pyrroles $A$ and $B$ are distinctly pyramidalized and there is a $\mathrm{NH}$... N hydrogen bond between pyrroles $D$ and $C$. For the $3-\mathrm{NO}_{2}$ substituted derivative, an increase in the dihedral angle between the tolyl plane and the macrocycle mean plane (taken as $19 \mathrm{C}+4 \mathrm{~N}$ ) up to $50.2^{\circ}$ was observed, resulting in enhanced macrocycle puckering. In the 3,17-diNO ${ }_{2}$-substituted derivative, this effect was even more pronounced. Optimized structures were shown to have very little sensitivity to the used functional and solvation effects.

Thus, from the data presented above, one can conclude that the molecular structure of free base corrole macrocycles has been understood in a qualitative manner. However, the presented data do not allow to make any quantitative comparison between the studied molecular structures, since most of them have been characterized on their own basis. The majority of cited papers deal with the quantification of the relative rotation of two adjacent pyrrole rings and treat this angle as the most indicative parameter for the molecular conformation. However, as indicated in the foregoing discussion, the way to determine this value has been quite different. Some values represent dihedral angles between the pyrrole planes (but no indication is given how these planes were defined and if the deviations from planarity of the pyrrole rings were taken into account), whereas other values represent the pyrrole tilting angles relative to the macrocycle mean plane (but the plane chosen was different). The easiest way toward a unified approach seems to be to use the sad-
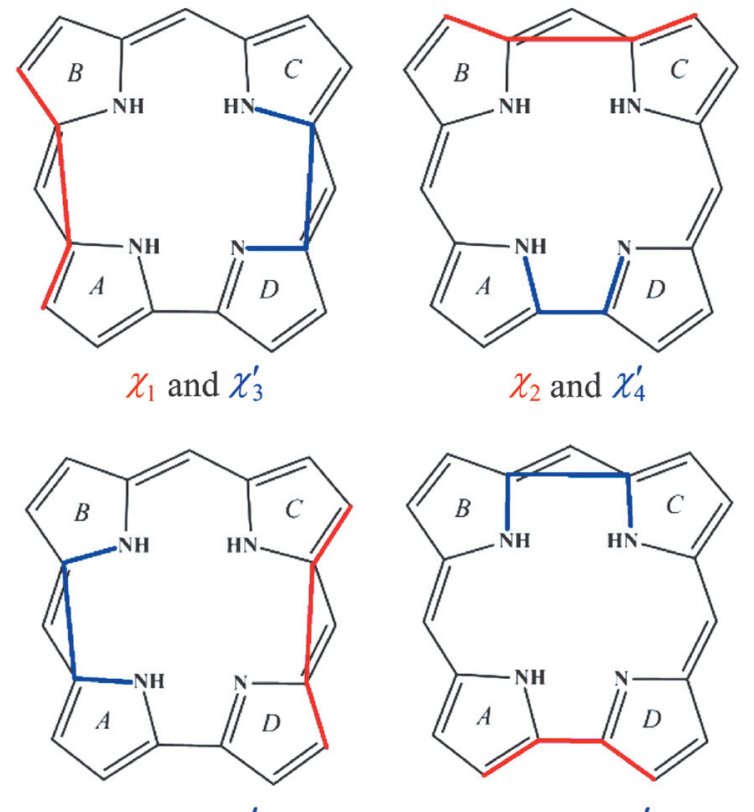

$\chi_{3}$ and $\chi_{1}^{\prime}$

$\chi_{4}$ and $\chi_{2}^{\prime}$ dling dihedrals $\chi_{\mathrm{i}}$ and $\chi_{\mathrm{i}}^{\prime}$ introduced by Ghosh et al. (Figure 3).${ }^{[17,18]}$ In most cases, the "outer" dihedral $\chi_{i}$ is sufficient, but when the pyrrole rings substantially deviate from planarity, the "inner" dihedral $\chi_{i}^{\prime}$ needs to be applied as well. ${ }^{[18]}$

Attempting to provide a unified data set on the mutual position of adjacent pyrrole rings, Ghosh et al. computed the dihedral angles $\chi_{i}$ for a series of free base corroles whose X-ray data were available. ${ }^{[18]}$ The calculated $\chi_{i}$ values were found to be completely different from the angle values reported in the original articles due to the different definitions used. Thus, one of the key structural elements of the corrole macrocycle can be determined in a unified way, but the question about the molecular conformation of the corrole macrocycle as a whole still needs to be addressed.

\section{Justification of the 7C Mean Plane and Analysis of the Macrocycle Conformations for Two Corrole NH Tautomers}

As stressed in the Introduction, quantitative analysis of the macrocycle distortion depends on the choice of the reference plane. All the early approaches aiming to define a corrole macrocycle mean plane have two substantial shortcomings, as they do not take into account a) the intrinsically asymmetric character of the macrocycle distortions, and b) NH tautomerization. Due to the general asymmetry of the corrole structure, it is impossible to define the mean plane in a simple way, like it was done for porphyrins. Atoms are not equivalent with respect to their importance for the macrocycle plane definition. All the approaches used up to date deal with complete sets of elements of a given type: the $19 \mathrm{C}+4 \mathrm{~N}$ plane consists of all carbon and nitrogen atoms of the macrocycle, ${ }^{[6,10,11]}$ the $19 \mathrm{C}$ plane consists of all carbon atoms of the macrocycle, ${ }^{[13]}$ the $11 \mathrm{C}$ plane consists of all $\mathrm{C}_{\mathrm{a}}$ and $\mathrm{C}_{\mathrm{m}}$ atoms of the macrocycle, ${ }^{[11]}$ and the $4 \mathrm{~N}$ plane consists of the four pyrrole nitrogen atoms. ${ }^{[8]}$ The only documented case of an asymmetrical atom set was the analysis of the out-of-plane deviations of the core hydrogen atoms in $N$-aryl-substituted corroles, which has been done based on the $3 \mathrm{~N}$ plane of the unsubstituted pyrroles (i.e. those not $\mathrm{N}$-arylated) ${ }^{[9]}$

Based on the analysis of the optimized structures of both NH tautomers of 10-(4,6-dichloropyrimidinyl)5,15-dimesitylcorrole, we previously concluded that all the pyrrole nitrogen atoms and $\mathrm{C}_{\mathrm{b}}$ carbon atoms substantially deviate from the $11 \mathrm{C}$ plane formed by all other macrocycle carbon atoms. ${ }^{[4]}$ Thus, the $11 \mathrm{C}$ plane was estimated as the best approximation for a complete set macrocycle mean plane. However, it does not explicitly consider the macrocycle asymmetry. On the other hand, 7 carbon atoms $\left(\mathrm{C}_{1}, \mathrm{C}_{4}\right.$, $\mathrm{C}_{5}, \mathrm{C}_{6}, \mathrm{C}_{9}, \mathrm{C}_{16}, \mathrm{C}_{19}$ ) remain practically in the same plane for both $\mathrm{NH}$ tautomers, whereas the two $\mathrm{C}_{\mathrm{a}}$ atoms of pyrrole $C$ $\left(\mathrm{C}_{11}\right.$ and $\left.\mathrm{C}_{14}\right)$ and the two adjacent $\mathrm{C}_{\mathrm{m}}$ carbons of the methine bridges $\left(\mathrm{C}_{10}\right.$ and $\left.\mathrm{C}_{15}\right)$ significantly deviate from this plane. The magnitude of these deviations was found to differ noticeably among the two NH tautomers. Thus, the 7C plane (Figure 4), given by the least-square distances to carbon atoms $\mathrm{C}_{1}, \mathrm{C}_{4}, \mathrm{C}_{5}, \mathrm{C}_{6}, \mathrm{C}_{9}, \mathrm{C}_{16}$, and $\mathrm{C}_{19}$, is suggested to be a better choice than the $11 \mathrm{C}$ plane. ${ }^{[4]}$ This mean plane, being the same for both NH tautomers, enables structural analysis

Figure 3. Definition of saddling dihedrals $\chi_{\mathrm{i}}$ and $\chi_{\mathrm{i}^{\prime}}{ }^{[18]}$ 


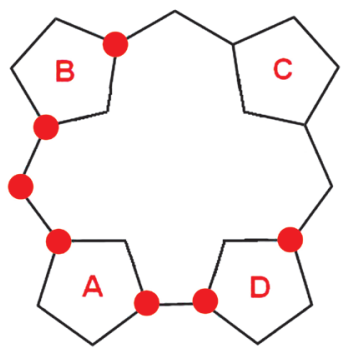

Figure 4. Corrole structure indicating the skeletal atoms defining the $7 \mathrm{C}$ mean plane

of the corrole NH tautomers in the same frame. Due to high rate constant of tautomer interconversion, the equilibrium averaged structure needs to be considered with the same invariant mean plane. ${ }^{[12]}$

However, the amplitude of the deviations of the individual atoms of the mean plane did not allow quantifying the type and magnitude of nonplanar macrocycle distortions as a whole. To characterize the nonplanar distortions of the contracted corrole macrocycle containing 23 skeletal atoms, the $\Delta 23$ value has been introduced, as was done earlier for porphyrins (i.e. $\Delta 24$ value):

$$
\Delta 23=\sqrt{\frac{1}{23} \sum_{i=1}^{23} \Delta z_{i}^{2}},
$$

where $\Delta z_{i}$ is the deviation for the $i$-th atom of the macrocycle from the macrocycle mean $7 \mathrm{C}$ plane. ${ }^{[19]}$

Both DFT-optimized structures of the 10-(4,6-dichloropyrimidinyl)-5,15-dimesitylcorrole tautomers exhibit equal nonplanar distortions, with $\Delta 23$ values of 0.200 and $0.198 \AA$ for the T1 and T2 tautomer, respectively. ${ }^{[19]}$ Analysis of the X-ray determined structure of the T1 tautomer of 2-nitro-5,10,15-tritolylcorrole ${ }^{[6]}$ revealed almost the same value, $\Delta 23=0.215 \AA$. The minor increase of the $\Delta 23$ value for the latter was suggested to be the result of the difference in the rotational degree of freedom of the aryl substituents on the meso-positions, which is higher in the latter case due to the lack of ortho-substituents on the aryl rings.

Analysis of the diagrams shown in Figure 5 indicates that the asymmetric character of the nonplanar distortions for both corrole NH tautomers is a characteristic feature of the free base corrole molecular conformation. Thus, the corrole macrocycle distortion pattern (Figure 5a-c) is similar to that of a porphyrin macrocycle in which bulky peripheral substituents are localized asymmetrically at one side of the macrocycle (Figure 5d). ${ }^{[2]}$ The porphyrin molecule forms a saddle-type conformer in which all the nitrogen atoms are in the macrocycle mean plane and tilted alternatively up and down relative to the porphyrin mean plane. ${ }^{[20,21]}$ On the contrary, in the case of corroles, the nitrogen atoms of pyrrole rings $A, C$ and $D$ are tilted out of the macrocycle mean plane in the same direction, and only pyrrole $B$ tilts in the opposite direction. Such molecular conformation is characterized by a wave-like distortion. It is important to mention that the direction of the wave-like distortion remains unchanged during $\mathrm{NH}$ tautomerization. ${ }^{[4,19,22]}$ The

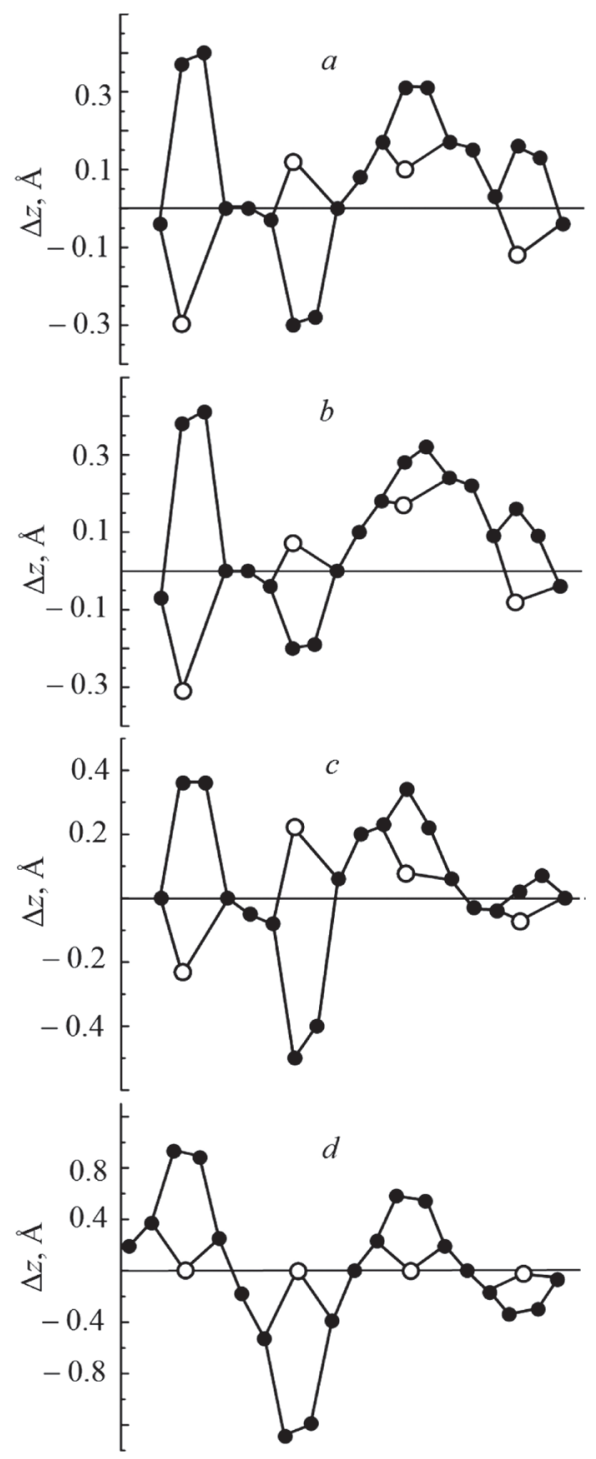

Figure 5. Diagram of atom deviations from the macrocycle mean plane: $a, b-\mathrm{T} 1$ and $\mathrm{T} 2$ tautomers of 10-(4,6-dichloropyrimidinyl)-5,15-dimesitylcorrole; $c$ - T1 tautomer of 2-nitro-5,10,15-tritolylcorrole; $d$-2,3,7,8-tetraethyl-5,10,15,20-tetraphenylporphyrin (atom coordinates taken from Ref. ${ }^{[19]}$ ). Pyrrole rings $A, B, C$ and $D$ are counted from left to right.

formation of "classical" alternate tilting of the pyrrole rings in free base corroles, as occurs in case of free base porphyrins, is prevented by the inner NH...N hydrogen bonding, providing some driving force for macrocycle planarization.

The general type of nonplanar distortion for both NH tautomers can be assigned as a wave-like distortion, with the wave axis going through the pyrroles containing $\mathrm{N}_{22}$ and $\mathrm{N}_{24}$, denoted in Figure 1 as pyrroles $B$ and $D$, respectively. Pyrroles $A$ and $C$, containing $\mathrm{N}_{21}$ and $\mathrm{N}_{23}$, have a substantially different orientation with respect to the wave axis. As noticed above, this distortion seems to originate from the trend toward coplanarization of pyrroles $C$ and $D$ due to NH...N hydrogen bonding. On the other hand, it may also increase the dihedral angle between pyrroles $D$ and $A$. Therefore, the term wave distortion should be considered within the framework introduced by Smith et al., ${ }^{[10]}$ imply- 
ing the need to take into account the asymmetry distinguishing the structure of corroles and porphyrins in more than only one meso-carbon atom. Due to this asymmetry, there is some arbitrariness in the direction of the wave axis. However, analysis of available macrocycle structures of both tautomers allows to conclude that pyrrole $B$ is the most symmetrical element. Thus, the wave axis was assigned to pass pyrrole $B$ through the $\mathrm{N}_{22}$ nitrogen atom, bisecting the $\mathrm{C}_{7}-\mathrm{C}_{8}$ bond. ${ }^{[4]}$

One more characteristic feature of the free base corrole macrocycle was found to be the substantial deviation of the positions of the pyrrole hydrogen atoms from the respective pyrrole planes. ${ }^{[4,8,19]}$ These displacements, which correspond to the pyramidalization of the pyrrole nitrogen atoms, imply that they acquire some $s p^{3}$ character. A raw estimation of this mixed hybridization $s p^{\lambda^{2}}$ was done with the equation $1+\lambda^{2} \cos \theta=0,{ }^{[23]}$ where $\theta$ is the mean angle for the $\mathrm{C}-\mathrm{N}-\mathrm{C}$ and two $\mathrm{H}-\mathrm{N}-\mathrm{C}$ bonds, considering that all three angles $\left(\mathrm{C}_{\mathrm{a}}-\mathrm{N}-\mathrm{C}{ }_{\mathrm{a}}, \mathrm{C}_{\mathrm{a}}-\mathrm{N}-\mathrm{H}\right.$ and $\left.\mathrm{C}_{\mathrm{a}}{ }_{\mathrm{a}}-\mathrm{N}-\mathrm{H}\right)$ are different. Thus, the maximum pyramidalization was found for pyrrole $B$ of the T1 tautomer of 10-(4,6-dichloropyrimidinyl)5,15 -dimesityl-corrole, with a value $\lambda^{2}=2.19 .{ }^{[4]}$ The pyramidalization patterns are clearly different for the two $\mathrm{NH}$ tautomers. For the above case, pyrrole $B$ has a maximum value of the angle $\delta$ between the $\mathrm{N}-\mathrm{H}$ bond and the pyrrole ring plane of about $26^{\circ}$, whereas the $\delta$ values for pyrroles $A$ and $C$ are 4.7 and $3.1^{\circ}$, respectively. Similar $\delta$ values of 8.6, 21.9 and $2.7^{\circ}$ have been calculated for pyrroles $A, B$ and $C$ of the T1 tautomer of 2-nitro-5,10,15-tritolylcorrole. ${ }^{[19]}$ For the T2 tautomer of 10-(4,6-dichloropyrimidinyl)-5,15-dimesitylcorrole, the maximum $\delta$ value was found to be lower, but the values for all three pyrroles are of about the same order of magnitude: $18.9^{\circ}$ for $A, 15.7^{\circ}$ for $B$ and $6.7^{\circ}$ for $D$, respectively. ${ }^{[19]}$

The significant out-of-plane displacement of the pyrrole protons has been noticed for the first time by Gross et $a l .,{ }^{[8]}$ and the degree of displacement has been suggested to relate to the macrocycle acidity, i.e. the higher the pyramidalization, the higher the acidity of a given corrole macrocycle. The differences in pyramidalization of the pyrrole nitrogen atoms for the two tautomers of 10-(4,6-dichloropyrimidinyl)-5,15-dimesitylcorrole were used to account for the experimentally observed acidity difference between these tautomers. ${ }^{[3]}$ It was concluded that the lower pyramidalization for the three pyrrole nitrogen atoms of the $\mathrm{T} 2$ tautomer is more important than the higher pyramidalization for one pyrrole nitrogen atom of the T1 tautomer, since the $\mathrm{T} 2$ tautomer is most acidic, ${ }^{[4]}$ i.e. any of the three pyrrolic protons in the corrole macrocycle core is able to undergo dissociation under basic conditions.

\section{Relationship between Peripheral Substitution Architecture and Macrocycle Conformation}

Sterical hindrance due to peripheral macrocycle substitution is known to add marginally to the magnitude of nonplanar distortions. ${ }^{[24]}$ Sterically unhindered 5,10,15,20-tetraphenylporphyrin has a $\Delta 24$ value of $0.05 \AA$, which grows up to $0.54 \AA$ for dodecasubstituted $2,3,7,8,12,12,17,18$-octabromo-5,10,15,20-tetraphenyl-porphyrin. ${ }^{[20,24]}$ Therefore, it is reasonable to consider the nonplanar distortions of tris- meso-aryl substituted corroles amounting to a $\Delta 23$ value of about $0.2 \AA$ as those due to intrinsic corrole macrocycle strains. One can expect that loading of the free base corrole macrocycle with bulky peripheral substituents will increase the magnitude of the nonplanar distortions. Full macrocycle substitution in 2,3,7,8,12,12,17,18-octabromo-5,10,15-trispentafluorophenylcorrole has been studied with both X-ray analysis (the T1 tautomer was revealed in the crystal) and dispersion-corrected DFT quantum-chemical calculations. ${ }^{[18]}$ The degree of distortion has been evaluated with calculations of the dihedral angles $\chi_{i}$. It was found that the $\chi_{1}$ value amounts to $100.4^{\circ}$, compared to $56.1^{\circ}$ in the archetype unsubstituted free base corrole, $68.7^{\circ}$ for 5,10,15-trispentafluorophenylcorrole and $79.4^{\circ}$ for 5,10,15-triphenylcorrole, respectively. The calculated $\Delta 23$ value for the crystal structure was found to increase more than two times, up to $0.503 \AA$, for the dodecasubstituted corrole compared to the tris-meso-aryl substituted derivatives. ${ }^{[25]}$ This value almost reaches the limiting $\Delta 24$ value reported for dodecasubstituted porphyrin (see above).

One can expect that the overall flexibilities of the free base corrole and porphyrin macrocycles are very similar, but in the case of corroles, two-fifth ( $c a .0 .2 \AA)$ of the $\Delta 23$ value is exhausted by means of the intrinsic strains in the corrole macrocycle and the remaining part ( $c a .0 .3 \AA$ ) is due to the peripheral substitution strains. In porphyrins, the total $\Delta 24$ value can be achieved by means of peripheral substitution effects.

Due to the contraction of the corrole macrocycle, the methine bridge $\left(\mathrm{C}_{4} \mathrm{C}_{5} \mathrm{C}_{6}\right.$ and $\left.\mathrm{C}_{14} \mathrm{C}_{15} \mathrm{C}_{16}\right)$ angles decrease for $3-5^{\circ},{ }^{[1,26]}$ giving little more space to neighboring pyrrole ring substituents, thus decreasing the limiting strains and making these strains more localized on the separate pyrroles. On the contrary, the steric interaction of two substituents attached to the $\mathrm{C}_{2}$ and $\mathrm{C}_{18}$ atoms would increase due to the shorter distance between them. Therefore, these two substituents (when present) would play a somewhat specific role for the macrocycle conformation. ${ }^{[26]}$ Depending on the substitution pattern, the sterical strain relaxes either with nonplanar distortion (increase in the dihedral $\chi_{4}$ value) or with in-plane distortion (pyrroles $A$ and $D$ rotations, increasing the distance between $\mathrm{C}_{2}$ and $\mathrm{C}_{18}$, and, simultaneously, decreasing the distance between $\mathrm{N}_{21}$ and $\mathrm{N}_{24}$; opposed motion of the two pyrroles $A$ and $D$ along the $\mathrm{C}_{1}-\mathrm{C}_{19}$ bond, thus increasing its length). Thus, the $\mathrm{C}_{1}-\mathrm{C}_{19}$ bond length becomes a sensitive structural parameter. ${ }^{[26]}$

It is worth to point out that pronounced pyrrole nitrogen pyramidalization, as described in the previous section, is also observed for derivatives with a high degree of nonplanar distortion. ${ }^{[25,26]}$ Thus, $\delta$ values of 5.0, 23.9 and $2.3^{\circ}$ have been calculated for pyrroles $A, B$ and $C$ of $\quad 2,3,7,8,12,12,17,18$-octabromo-5,10,15-tris-pentafluorophenylcorrole. ${ }^{[25]}$ These values are of the same order of magnitude as for tris-meso-aryl-substituted derivatives, ${ }^{[19]}$ indicating the absence of a direct relationship with the magnitude of nonplanar distortions. This behavior is similar to that observed for the $N$-alkylated derivatives of porphyrins, where the pyramidalization remains upon going from the free bases to the saddle-type distorted doubly protonated species, for which the sterical hindrance in the macrocycle core decreases. ${ }^{[27,28]}$ 


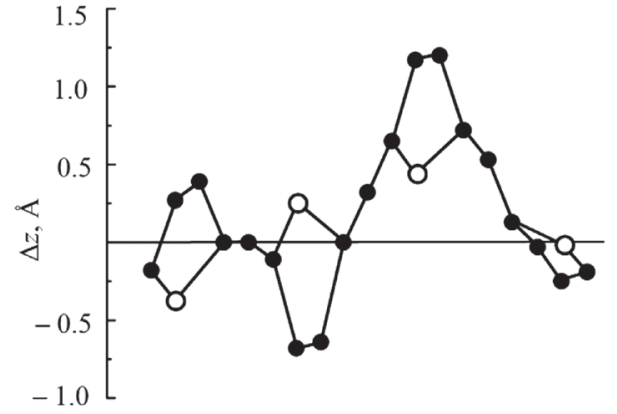

Figure 6. Diagram of atom deviations from the macrocycle mean plane for the T1 tautomer of 2,3,7,8,12,12,17,18-octabromo5,10,15-tris-pentafluorophenylcorrole (atom coordinates taken from Ref.[18]).

Let's then turn to the analysis of the diagram of atom deviations from the mean plane (Figure 6). ${ }^{[25]}$ An important conclusion from literature ${ }^{[19,25]}$ is that the molecular conformation in the case of high macrocycle distortion is of the saddle-type, in contrast with the wave-type observed for less distorted corrole derivatives. Thus, an increase in the magnitude of nonplanar distortions leads to a wave-to-saddle conformational transition of the free base corrole macrocycle.

The macrocycle conformations for a family of 18 methylated free base corrole derivatives (as T1 tautomers) have recently been optimized by quantum-chemical methods to study this phenomenon in more detail. ${ }^{[26,29]}$ The number of the attached methyl groups and the substitution architecture determine the character and the degree of the nonplanar macrocycle distortions, and the $s p^{3}$-hybridized methyl carbon atoms are expected to provide high steric strains. It was found that all the studied compounds can be divided into four groups based on the $\Delta 23$ value. The first group with minimal $\Delta 23$ values of $0.267-0.294 \AA$ consists of the compounds where the steric hindrance localizes on the separate pyrrole rings. Then, $\Delta 23$ values ranging from 0.304 to $0.326 \AA$ are reported for those compounds where one or two (with no interaction between them) sterically hindered domains consisting of two pyrrole rings are formed. Values for $\Delta 23$ between 0.377 and $0.380 \AA$ are found for those macrocycles where one sterically hindered domain includes all three meso-positions of the macrocycle. Finally, the undecasubstituted corrole, where all eleven methyl groups form one sterically hindered annular domain, represents the fourth and final group, with a maximum $\Delta 23$ value of $0.413 \AA{ }^{\left[{ }^{[26]}\right.}$ Saddle-type nonplanar distortions of the macrocycle are shown to take place for two derivatives with extremely high $\Delta 23$ values $(0.380$ and $0.413 \AA)$. The macrocycles with $\Delta 23$ values of $0.304-0.326 \AA$ are characterized as boundary ones. In these compounds, one pyrrole ring lies practically in the $7 \mathrm{C}$ plane. The other derivatives with smaller $\Delta 23$ values show a wave-type nonplanar distortion of the macrocycle. ${ }^{[29]}$ The dihedral angles $\chi_{1}$ and $\chi_{2}$ parallel the $\Delta 23$ parameter trend, but $\chi_{3}$ and $\chi_{4}$ do not and reflect the specific sterical strains induced by substitution. The distance between the nitrogen atoms of pyrroles $A$ and $C$ also parallels the $\Delta 23$ value and leads to an increase in the macrocycle asymmetry with rising nonplanar distortion. The $\mathrm{C}_{1}-\mathrm{C}_{19}$ bond length in the dipyrrole unit reflects the local specific interactions rather than the overall macrocycle distortion, as was stressed above, and varies in the range of $1.420-1.434 \AA .^{[26]}$

\section{Conformational Dynamics of the Corrole Macrocycle}

The electronic ground states of two corrole NH tautomers were shown to have similar macrocycle conformations ${ }^{[4,18]}$ However, tautomerization triggers conformational changes to allow the macrocycle core to accommodate another configuration of the protons. The type of macrocycle distortion remains the same for the two $\mathrm{NH}$ tautomers, ${ }^{[4,18]}$ but the out-of-plane atom displacements are susceptible to rearrangement. The early suggestion on the relative flatness of the potential energy surfaces associated with outof-plane distortions ${ }^{[5]}$ has been confirmed more recently by DFT calculations. ${ }^{[18]}$ Thus, it was found that regardless of the specific substitution pattern, $\chi_{1}$ and $\chi_{2}$ dihedral variations of $\pm 20^{\circ}$ take place at the expense of no more than $1 \mathrm{kcal} / \mathrm{mol}$, while the barrier for tautomerization is about $5 \mathrm{kcal} / \mathrm{mol} .^{[18]}$ This last estimation fits well to the experimentally measured value of $4.3 \mathrm{kcal} / \mathrm{mol}$ for the tautomerization of 10-(4,6-dichloropyrimidinyl)-5,15-dimesitylcorrole in the singlet excited $\left(\mathrm{S}_{1}\right)$ state. ${ }^{[30]}$

In contrast with the electronic ground $\left(\mathrm{S}_{0}\right)$ state, in which the type and degree (as indicated by the $\Delta 23$ value) of nonplanar distortions for the two NH tautomers of 10-(4,6-dichloropyrimidinyl)-5,15-dimesitylcorrole are very similar, ${ }^{[4,19]}$ the two corrole $\mathrm{NH}$ tautomers differ dramatically in the lowest excited triplet $\left(\mathrm{T}_{1}\right)$ state. ${ }^{[29]}$ The T1 tautomer has a clear signature of a wave-type macrocycle conformation, whereas the T2 tautomer demonstrates a boundary structure with pyrrole $D$ showing a barely visible tilt in the direction opposed to that of pyrrole $B$. The $\Delta 23$ values are 0.330 and $0.246 \AA$ for the $\mathrm{T} 1$ and $\mathrm{T} 2$ tautomers, respectively. Both these values are distinctly higher than the values determined for the electronic ground state (see above), indicating an increase in the out-of-plane distortion when the lowest excited triplet state populates. The T1 tautomer has a substantially higher deviation from macrocycle planarity (Figure 7).

The $\mathrm{C}_{1}-\mathrm{C}_{19}$ bond length in the lowest excited triplet state increases up to $1.452-1.454 \AA \AA^{[29]}$ Taking into account the simultaneous increase in the $\Delta 23$ value, one can conclude that both the out-of-plane and in-plane macrocycle distortions increase when the free base corrole is excited to the triplet manifold. The excitation energy dissipation is followed by conformational relaxation of the macrocycle, which feeds back to the electronic structure of the molecule, resulting in changes of the excited state energy and lifetime. [31] Thus, these conformational dynamics need to be taken into account upon analysis of the lowest excited triplet state deactivation. Thus, deactivation of the $\mathrm{T}_{1}$ state to the singlet ground state can be considered as the transition between two wave-type molecular conformations for the $\mathrm{T} 1$ tautomer, whereas the $\mathrm{T} 2$ tautomer needs to undergo a conformational transition in order to deactivate, since its $\mathrm{T}_{1}$ state conformation is not "purely" wave-type. 

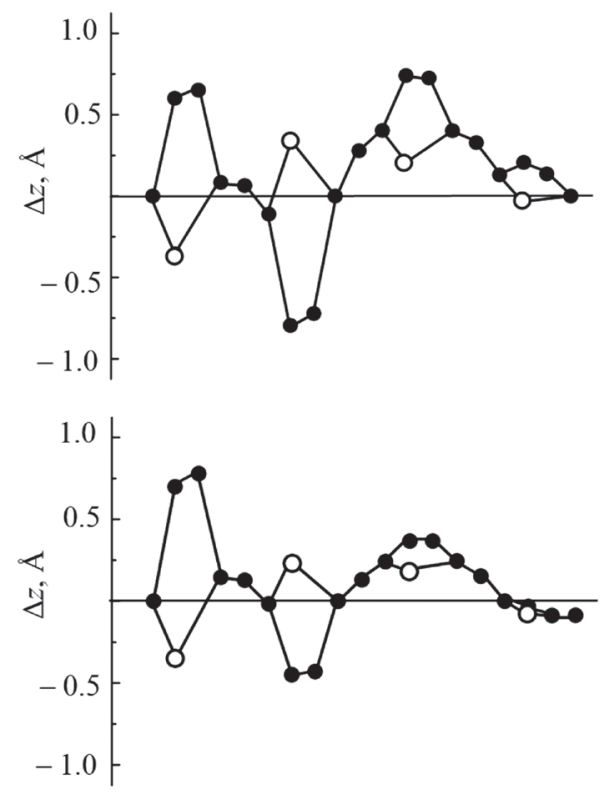

Figure 7. Diagram of atom deviations from the macrocycle mean plane for the T1 (top) and T2 (bottom) tautomers of 10-(4,6-dichloropyrimidinyl)-5,15-dimesitylcorrole in the lowest excited triplet $\left(\mathrm{T}_{1}\right)$ state (atom coordinates taken from Ref. ${ }^{[29]}$ ).

One more source of conformational dynamics of free base corroles needs to be considered. The free base corrole macrocycle is asymmetric and lacks a center of inversion, which gives rise to the possible formation of enantiomers. Pointed out almost half a century ago in the pioneering X-ray study by Hodgkin et al. (in 1971), ${ }^{[1]}$ this interesting feature has been out of the scope of researchers. In fact, we only know of one single paper dealing with this subject. ${ }^{[18]}$ Ghosh et al. computed the potential energy surfaces as a function of "inner" dihedral $\chi_{1}^{\prime}$ for both $\mathrm{NH}$ tautomers of 2,3,7,8,12,12,17,18-octabromo-5,10,15-trispentafluorophenylcorrole. An abrupt inversion of the macrocycle structure upon formation of the other enantiomer was found when the dihedral $\chi_{1}^{\prime}$ amounted $\pm 28^{\circ}$ and $\pm 26^{\circ}$ for the T1 and T2 tautomer, respectively. The energy barrier for enantiomerization was found to be about $15 \mathrm{kcal} / \mathrm{mol}$. These figures, taken together with the energy of the $\mathrm{NH}$ tautomerization barrier, allow to point out a hierarchy of macrocycle conformational dynamics in the electronic ground state. At normal conditions ( $k T$ about $0.6 \mathrm{kcal} / \mathrm{mol}$ ), $\mathrm{NH}$ tautomerization always takes place within a given enantiomer structure. However, when the excited states are populated, both $\mathrm{NH}$ tautomerization and enantiomerization can proceed due to the excess of energy. This results in a different photoresponse and/or excited state reactivity of free base corroles.

\section{Conclusions}

Free base corroles constitute a unique family of macrocyclic compounds, possessing a large molecular flexibility and the capacity for conformational rearrangements, includ- ing NH tautomerization, enantiomerization and excited state conformational relaxation. Proper interpretation of the molecular structure is the basis for the correct understanding of the reactivity, spectral-luminescent and photophysical properties of these intriguing compounds. The presented review summarizes the available results on the molecular structure of free base corroles and provides an analysis in terms of the recently introduced $7 \mathrm{C}$ mean plane, taking into account the intrinsic molecular asymmetry and NH tautomerization. The authors have aimed to tackle the challenge of comparing data from different research groups, which was nearly impossible so far due to the use of different basics. This work therefore presents the first comprehensive viewpoint on the conformation of free base corrole macrocycles.

Acknowledgements. W. Maes acknowledges the Research Foundation - Flanders (FWO) and Hasselt University for financial support. M. Kruk and D. Klenitsky acknowledge the State Program of Scientific Researches of the Republic of Belarus "Photonics, opto- and microelectronics", grant no. 1.3.01 for financial support.

\section{References}

1. Harrison H.R., Hodder O.J.R., Hodgkin D.C. J. Chem. Soc. (B) 1971, 640-645.

2. Shelnutt J.A., Song X.-Z., Ma J.-G., Jia S.-L., Jentzen W., Medforth C.J. Chem. Soc. Rev. 1998, 27, 31-41.

3. Ivanova Yu.B., Savva V.A., Mamardashvili N.Zh., Starukhin A.S., Ngo T.H., Dehaen W., Maes W., Kruk M.M. J. Phys. Chem. A 2012, 116, 10683-10694.

4. Beenken W.J.D., Presselt M., Ngo T.H., Dehaen W., Maes W., Kruk M.M. J. Phys. Chem. A 2014, 118, 862-871.

5. Ghosh A., Junge K. Chem. Eur. J. 1997, 3, 823-833.

6. Stefanelli M., Pomarico G., Tortora L., Nardis S., Fronczek F.R., McCandless G.T., Smith K.M., Manowong M., Fang Y., Chen P., Kadish K.M., Rosa A., Ricciardi G., Paolesse R. Inorg. Chem. 2012, 51, 6928-6942.

7. Gross Z., Galili N., Simkhovich L., Saltsman I., Botoshansky M., Bläser D., Boese R., Goldberg I. Org. Lett. 1999, 1, 599-602.

8. Simkhovich L., Goldberg I., Gross Z. J. Inorg. Biochem. 2000, 80, 235-238.

9. Simkhovich L., Paramesvar I., Goldberg I., Gross Z. Chem. Eur. J. 2002, 8, 2595-2601.

10. Paolesse R., Marini A., Nardis S., Froiio A., Mandoj F., Nurco D.J., Prodi L., Montalti M., Smith K.M. J. Porphyrins Phthalocyanines 2003, 7, 25-36.

11. Paolesse R., Nardis S., Venanzi M., Mastroianni M., Russo M., Fronczek F.R., Vicente M.G.H. Chem. Eur. J. 2002, 9, 1192-1197.

12. Balasz I.S., Saltsman I., Mahammed A., Tkachenko E., Golubkov G., Levine J., Gross Z. Magn. Reson. Chem. 2004, 42, 624-635.

13. Ding T., Harvey J.D., Ziegler C.J. J. Porphyrins Phthalocyanines 2005, 9, 22-27.

14. Gao Di, Canard G., Giorgi M., Balaban T.S. Eur. J. Inorg. Chem. 2012, 36, 5915-5920.

15. Costa R., Richard Geier III G., Ziegler C.J. Dalton Trans. 2011, 40, 4384-4386.

16. Fujino K., Hirata Y., Kawabe Y., Moromoto T., Srinivasan A., Toganoh M., Miseki T., Kudo A., Furuta H. Angew. Chem. Int. Ed. 2011, 50, 6855-6859. 
17. Thomas K.E., Alemayehu A.B., Conradie J., Beavers C.M., Ghosh A. Acc. Chem. Res. 2012, 45, 1203-1214.

18. Capar J., Conradie J., Beavers C.M., Ghosh A. J. Phys. Chem. A 2015, 119, 3452-3457.

19. Klenitsky D.V., Kruk M.M. Proceedings of Belarusian State Technological University (BSTU) [Trudy BGTU ], no. 4: Chem., Technol. Org. Subst. and Biotechnol. 2015, 177, 20-23.

20. Röder B., Büchner M., Rückmann I., Senge M.O. Photochem. Photobiol. Sci. 2010, 9, 1152-1158.

21. Senge M.O. Chem. Commun. 2006, 243-256.

22. Klenitsky D.V., Kruk M.M. Proceedings of Belarusian State Technological University (BSTU) [Trudy BGTU], no. 6: Phys. Math. and Comp. Sci. 2014, 170, 44-46.

23. Petrucchi R.H., Harwood W.S., Herring F.G. General Chemistry. Principles and Modern Applications, $8^{\text {th }}$ ed., PrenticeHall: Englewood Cliffs, NJ, 2002. p. 1-441.

24. Senge M.O. Highly Substituted Porphyrins. In: The Porphyrin Handbook, Vol. 1 (Kadish K.M., Smith K.M., Guillard R., Eds.) San Diego: World Scientific, 2000. p. 239-347.
25. Klenitsky D.V., Kruk M.M., Maes W. Proceedings of Belarusian State Technological University (BSTU) [Trudy BGTU ], Ser. 2, Chem. Engin., Biotechn., Geoecol., no. 1, 2017, $23-28$.

26. Kruk M.M., Klenitsky D.V., Maes W. Proceedings of Belarusian State Technological University (BSTU) [Trudy BGTU ], Ser. 3, Phys. Math. and Comp. Sci., no. 1, 2018, 36-42.

27. Broadhurst M.J., Grigg R., Shelton G. Chem. Commun. 1970, 231-233.

28. Jackson A.H., Deraden G.R. Ann. N. Y. Acad. Sci. 1973, 206, 151-175.

29. Kruk M.M., Klenitsky D.V., Gladkov L.L., Maes W. Proceedings of Belarusian State Technological University (BSTU) [Trudy BGTU ], Ser. 3, Phys. Math. and Comp. Sci., no. 1, 2019, 6-13.

30. Ajeeb Y., Karlovich T., Maes W., Dehaen W., Kruk M.M. In: ICPP-10, Book of Abstracts. Munich, Germany, July 1-7, 2018, p. 398.

31. Knuykshto V.N., Ngo T.H., Dehaen W., Maes W., Kruk M.M. RSC Adv. 2016, 6, 43911-43915. 